\title{
Evaluation of laparoscopic vs robotic partial nephrectomy using the margin, ischemia and complications score system: A retrospective single center analysis
}

\author{
Stefano Ricciardulli ${ }^{1,2}$, Qiang Ding ${ }^{1}$, Xu Zhang ${ }^{1}$, Hongzhao Li ${ }^{1}$, Yuzhe Tang ${ }^{1}$, Guoqiang Yang ${ }^{1}$, \\ Xiyou Wang ${ }^{1}$, Xin Ma ${ }^{1}$, Alberto Breda ${ }^{3}$, Antonio Celia ${ }^{2}$ \\ ${ }^{1}$ Department of Urology, Chinese PLA General Hospital, Beijing, China; \\ ${ }^{2}$ Department of Urology, San Bassiano Hospital, Bassano Del Grappa, Italy; \\ ${ }^{3}$ Department of Urology, Foundacio Puigvert Universidad Autonoma de Barcelona, Spain.
}

\begin{abstract}
Summary Objective: To evaluate differences between Laparoscopic Partial Nephrectomy (LPN) and Robot-Assisted Partial Nephrectomy (RAPN) using the Margin, Ischemia and Complications (MIC) score system and to evaluate factors related with MIC success.

Materials and Methods: Single centre retrospective study on 258 LPN and 58 RAPN performed between January 2012 and January 2014. Success was defined when surgical margins was negative, Warm Ischemia Time (WIT) was $\leq 20$ minutes and no major complications occurred. Mann-Whitney-U and Pearson $X 2$ correlation were used to compare LPN and RAPN. A matched pair comparison was also performed. Spearman correlation (Rho) was used to evaluate the relationship between clinical, intra and post-operative and pathological patients characteristics with MIC score. A binary regression analysis was also performed to evaluate independent factors associated with MIC success.
\end{abstract}

Results: The MIC rate in LPN and RAPN was 55\% and $65.5 \%$ respectively. No differences in clinical, intra and post-operative outcomes between groups were found. Clinical tumor size ( $p$-value: < 0.001; OR: 0.829; 95\% CI: 0.697-0.987), PADUA score ( $p$-value: < 0.001; OR: 0.843; 95\% CI: 0.740-0.960), PADUA risk groups (intermediate; p-value: < 0.001; OR: 0.416; 95\% CI: 0.2380.792; high: $p$-value: < 0.001; OR: 0.356; 95\% CI: 0.199 0.636), WIT (p-value: < 0.001; OR: 0.598; 95\% CI: 0.530$0.675)$ were independently associated with MIC. eGFR (<60 vs $\geq 60 \mathrm{ml} / \mathrm{min}$ per $1.73 \mathrm{~m}^{2}$ : p-value: $<0.001$; OR: 3.356; 95\% CI: 1.701-6.621) and Fuhrman nuclear grade (p-value: 0.014; OR: 1.798; 95\% CI:1.129-2.865) were also independently associated with MIC.

Conclusions: MIC score system is a simple and useful tool to report and to compare different surgical approach.

KEY WORDS: Complications; Laparoscopic partial nephrectomy; Positive margins; Robot-assisted partial nephrectomy; Warm ischemia time.

Submitted 5 September 2014; Accepted 31 December 2014

\section{INTRODUCTION}

International guidelines on Renal Cell Carcinoma (RCC), states that renal tumors $\leq 7 \mathrm{~cm}$ are best managed by nephron sparing surgery (NSS) $(1,2)$. Open partial nephrectomy (OPN) represents the gold standard for renal tumors $\leq 7 \mathrm{~cm}$, while laparoscopic partial nephrectomy (LPN) and Robot-Assisted Partial Nephrectomy (RAPN) are the main alternatives. Partial nephrectomy (PN) is a more complex procedure and several aspects must to be evaluated (3). In recent years PN become a challenge procedure to have less Warm Ischemia Time (WIT), which represent the most important predictor of renal function after PN (4). As reported by some Authors (5) the best PN should ideally be without ischemia, but, when required, it should not exceed 20 minutes for warm ischemia and $<35$ minutes for cold ischemia. Ten years after the first case described by Gettman et al. (6), RAPN seems to be a promising procedure able to bridge the technical difficulties of LPN (7). RAPN has helped to reduce the surgical learning curve needed, and shortened operative and ischaemic times with less blood loss compared with LPN. In 2012 Buffi et al. (8), proposed a new score system to evaluate success in PN, the Margin, Ischemia and Complications (MIC). According to this newly proposed scoring system, an optimal PN is accomplished when surgical margins (SM) are negative, WIT was $\leq 20$ minutes and no major complication (9) (Clavien-Dindo grade 3-4) were observed. The use of this simple system could be of paramount importance to compare and evaluate different approach used to perform PN. Aim of this study is to evaluate difference between LPN and RAPN, from a single center experience, using the MIC score and to evaluate pre, intra and post-operative factors that may potentially influence this scoring system.

\section{Materials and Methods}

This is a retrospective single centre study approved by the local ethical committee. All patients were counselled about the risks, benefits and alternative treatments for 
the condition; individual informed consent was obtained. All patients that underwent LPN and RAPN performed by a single experienced surgeon between January 2012 and March 2014 were included in the analysis. Patients with solitary kidney, multifocal tumours, those with radiography evidence of metastases and PN performed with no ischemia time or cold ischemia were not considered suitable for the inclusion. LPN was performed with a retro-peritoneal approach as previously described (10-11) with renal artery clamping. The RAPN was performed using Da Vinci Si four-arm robot (Intuitive Surgical Inc, Sunnyvale, CA, USA) with standardized trans-peritoneal approach (7, 12-13) with renal artery clamping. From January 2012 to September 2014 all PNs were performed laparoscopically, after September robotically. To eliminate bias related to surgeon learning curve the first 30 RAPN (7) were eliminated from the database. Before surgery, all patients underwent a computed tomography (CT) scan or magnetic resonance imaging (MRI) in order to evaluate the clinical stage and the anatomical characteristics of the tumors. Based on image of CT scan or MRI, a Preoperative Aspect and Dimension Used for an Anatomical (PADUA) score (14) was assigned to each patients by two different examiners. Tumors were stratified into low-risk (PADUA score 6-7), intermediate-risk (PADUA score 8-9), and high-risk (PADUA score $\geq 10$ ) (14). The WIT and the estimated blood loss (EBL) were assessed by an anesthesiologist. Postoperative complications, occurred during the first 30 days after surgery, were classified according to the Dindo modification of the Clavien system $(9,15)$ and defined as minor (grade 1-2) and major (grade 3-4). Surgery duration was defined as the time from trocar placement to trocars removed for LPN and the console time for RAPN. Renal function was calculated by estimating the glomerular filtration rate (GFR) through the Modification of Diet in Renal Disease study (MDRD) formula preoperatively and post-operatively (16) using preoperative and latest postoperative (median 30 days after surgery) serum creatinine ( $\mathrm{sCr}$ ). Renal function was staged according to the National Kidney Foundation Disease Outcome Quality Initiative classification. Postoperative change in sCR and eGFR was also evaluated. An eGFR $<60 \mathrm{ml} / \mathrm{min}$ per $1.73 \mathrm{~m}^{2}$ was definited as an undesiderable event. The following information was available for each patient included in the study: age, BMI, ASA, Charlson comorbidity index (CCI), gender, clinical tumor size, PADUA score and PADUA anatomical features, WIT, surgery duration, EBL, conversion to open, intra and post-operative complications, pre and postoperative sCr. Pathological tumor size, histological subtypes according with the World Health Organization classification (17), tumor extension according with the TNM classification (18), nuclear grade according to the Fuhrman classification (19) and positive surgical margin rate were also reported. Positive surgical margins (PSM) were defined as the extension of the tumor over the inked parenchymal surface. According with the MIC score system success of procedures was defined when WIT was $\leq 20$ minutes, negative SM and no major grade of post-operative complications occurred (8). For this group of patients we assigned three points. If only two of these characteristics compared we assigned two points; only one of this characteristics, one point.

Descriptive statistics was performed. Continuous variables were reported as median and interquartile range (IQR); categorical variables were reported as number of cases (no) and percent (\%). A matched pair analysis was performed to adjust for preoperative using multivariable logistic regression on this covariates: clinical tumor size (continuous) and PADUA score (continuous and categorical). The matching was carried out with a $1: 1$ ratio (58 matched in LPN and 58 in RAPN) with respect to surgical approach (LPN vs RAPN). Non-parametric Mann-Whitney-U test was used to compare LPN and RAPN and matched LPN and RAPN with continuous variables, Pearson $\chi 2$ correlation was used for categorical variables. Spearman Rank Order Correlation (Rho) was used to evaluate the relationship between clinical, intra and post-operative and pathological patients characteristics with MIC score. The relationship was defined as small (rho $=0.10$ to 0.29 ), medium (rho $=0.30$ to 0.49 ) and large (rho $=0.50$ to 1 ). A binary regression analysis was done in order to evaluate the association between clinical, intra and post-operative and pathological characteristics with MIC. We used only factors statistically significant in Spearman analysis. A two side p-value of $<0.05$ was defined as statistically significant. All data were analyzed using SPSS v. 20 with Phyton extension (IBM Corp., Armonk, NY, USA).

\section{RESULTS}

A total of 316 patients were enrolled in this study (258 LPN vs 58 RAPN). Table 1 described patients' preoperative characteristics between LPN and RAPN. No difference was found between LPN and RAPN in demographic patients characteristics. The ASA score was higher in RAPN (pvalue: 0.041). Interestingly, the two groups presented the similar tumor characteristics, median size (3.1 [IQR: 2.13.8] vs 3.1 [IQR: 2.2-3.8]; p-value: 0.634), median PADUA score (8.5 vs 8.2; p-value: 0.306 ) and similar clinical stage (p-value: 0.487 ). According with PADUA score system, the PADUA risk groups presented a similar distribution between LPN and RAPN (low risk: $33.7 \%$ vs 32.8\%; intermediate risk: $34.9 \%$ vs $39.7 \%$; high risk: $31.4 \%$ vs $27.5 \%$; p-value: 0.765$)$. The only difference was founded in tumor face location, with a predominance of tumor located in the posterior face in LPN (63.2\% vs $36.2 \%)$ and a predominance of tumor located on anterior face in RAPN (63.8\% vs 36.8\%). No difference was found in preoperative sCr. $6.2 \%$ of patients in LPN (vs $1.7 \%$ in RAPN) had a eGFR level $<60 \mathrm{ml} / \mathrm{min}$ per $1.73 \mathrm{~m} 2$, but this was not statistically significative (p-value: 0.172 ). Table 2 showed intra, post-operative and pathological patients' characteristics. In this series we did not find any significant difference (p-value: 0.321 ) in WIT between LPN and RAPN (20.8 vs 19.4 minutes). WIT was $\leq 20$ minutes in $60.1 \%$ and $\geq 30$ minutes in $12.8 \%$ in LPN, while it was $65.5 \%$ and $5.2 \%$ in RAPN. No difference in intra and post-operative complications between LPN and RAPN was found. We reported a rate of intraoperative complications of $10.5 \%$ in LPN and $6.9 \%$ in RAPN. No conversion to OPN was observed in RAPN while 3 


\begin{tabular}{|c|c|c|c|c|c|}
\hline Variable & LPN $(n=258)$ & Matched $(n=58)$ & RAPN $(n=58)$ & P-value (full data set) & P-value (matched) \\
\hline Median age, years (IQR) & $49.3(41-58)$ & $48.4(41-56)$ & $51.6(45-59)$ & 0.174 & 0.142 \\
\hline Gender, no (\%) & & & & 0.172 & 0.108 \\
\hline Male & $169(65.5)$ & $36(62.1)$ & $44(75.9)$ & & \\
\hline Female & 89 (34.5) & $22(37.9)$ & $14(24.1)$ & & \\
\hline Median BMI, Kg/m² (IQR) & $25.3(22.4-27.5)$ & $25.4(20-25)$ & $25.6(21-25)$ & 0.834 & 0.842 \\
\hline Median ASA, no (IQR) & $1.8(2)$ & $1.8(2)$ & $1.9(2)$ & 0.041 & 0.052 \\
\hline Median CCI, no (IQR) & $0.55(0-1)$ & $0.5(0-1)$ & $0.5(0-1)$ & 0.439 & 0.562 \\
\hline Median clinical tumor size, cm (IQR) & $3.1(2.1-3.8)$ & $2.9(2-3.5)$ & $3.1(2.2-3.8)$ & 0.634 & 0.263 \\
\hline Clinical Stage, no (\%) & & & & 0.487 & 0.593 \\
\hline $\mathrm{T} 1 \mathrm{a}$ & $196(76)$ & $46(79.3)$ & $46(79.3)$ & & \\
\hline $\mathrm{T} 1 \mathrm{~b}$ & $56(21.7)$ & $11(19)$ & $12(20.7)$ & & \\
\hline T2a & $6(2.3)$ & $1(1.7)$ & $0(0)$ & & \\
\hline Median PADUA score, no (IQR) & $8.5(7-10)$ & $8.6(7-10)$ & $8.2(6-10)$ & 0.306 & 0.260 \\
\hline PADUA risk groups, no (\%) & & & & 0.765 & 0.892 \\
\hline Low (6-7) & $87(33.7)$ & $17(29.3)$ & $19(32.8)$ & & \\
\hline Intermediate (8-9) & $90(34.9)$ & $23(39.7)$ & $23(39.7)$ & & \\
\hline High $(\geq 10)$ & 81 (31.4) & $18(31)$ & $16(27.5)$ & & \\
\hline Side, no (\%) & & & & 0.178 & 0.135 \\
\hline Right & $135(52.3)$ & $28(48.3)$ & $36(62.1)$ & & \\
\hline Left & $123(47.7)$ & 30 (51.7) & $22(37.9)$ & & \\
\hline Face, no (\%) & & & & $<0.001$ & 0.003 \\
\hline Anterior & $95(36.8)$ & $21(36.2)$ & $37(63.8)$ & & \\
\hline Posterior & $163(63.2)$ & 37 (63.8) & $21(36.2)$ & & \\
\hline Median preoperative $\mathrm{sCr}, \mu \mathrm{mol} / \mathrm{L}(\mathrm{IQR})$ & $75.9(62-85)$ & $73.4(63-80)$ & $76.4(64-86)$ & 0.474 & 0.243 \\
\hline Median preoperative eGFR, $\mathrm{ml} / \mathrm{min}$ per $1.73 \mathrm{~m}^{2}$ (IQR) & $98.9(76-116)$ & $99.4(78-116)$ & $95.8(85-108)$ & 0.958 & 0.651 \\
\hline Preoperative eGFR < 60, no (\%) & $16(6.2)$ & $3(5.2)$ & $1(1.7)$ & 0.172 & 0.309 \\
\hline
\end{tabular}

Table 1.

Pre-operative patients characteristics.

LPN $=$ Laparoscopic Partial Nephrectomy; RAPN = Robot-Assisted Partial Nephrectomy; $I Q R=$ Interquartile Range; $B M I=$ Body Mass Index; $A S A=$ American Society of Anesthesiologist; IQR = Interquartile Range; PADUA $=$ Preoperative Aspects and Dimension Used for an Anatomical: $\mathrm{sCr}=$ serum Creatinine; eGFR = estimated Glomerular Filtration Rate.

\begin{tabular}{|c|c|c|c|c|c|}
\hline Variable & LPN $(n=258)$ & Matched $(n=58)$ & RAPN $(n=58)$ & P-value (full data set) & P-value (matched) \\
\hline \multicolumn{6}{|l|}{ Intra-operative characteristics } \\
\hline Median WIT, min (IQR) & $20.8(17-25)$ & $20.6(16-26)$ & $19.4(16-22)$ & 0.321 & 0.472 \\
\hline \% WIT, no (\%) & & & & 0.201 & 0.284 \\
\hline$\leq 20$ & $155(60.1)$ & $35(60.3)$ & $38(65.5)$ & & \\
\hline $21-29$ & $70(27.1)$ & 15 (25.9) & $17(29.3)$ & & \\
\hline$\geq 30$ & $33(12.8)$ & $8(13.8)$ & $3(5.2)$ & & \\
\hline Median surgery duration, $\min (\mathrm{IQR})$ & $112(90-130)$ & $110(87-150)$ & $114(90-120)$ & 0.385 & 0.372 \\
\hline Median EBL, ml (IQR) & $68(30-85)$ & $68(30-50)$ & $104(50-110)$ & $<0.001$ & 0.007 \\
\hline Intra-operative complications, no (\%) & $27(10.5)$ & $5(8.3)$ & $4(6.9)$ & 0.409 & 0.729 \\
\hline Conversion to OPN, no (\%) & $3(1.2)$ & $1(1.7)$ & $0(0)$ & 0.409 & 0.315 \\
\hline \multicolumn{6}{|l|}{ Post-operative complications } \\
\hline Post-operative complications, no (\%) & & & & 0.414 & 0.778 \\
\hline Minor, no (\%) & $44(17.1)$ & $14(24.1)$ & $15(25.9)$ & & \\
\hline Major, no (\%) & $2(0.8)$ & $0(0)$ & $1(1.7)$ & & \\
\hline Median hospital stay, days (IQR) & $7(7-8)$ & $7(7-8)$ & $7(7-8)$ & 0.975 & 0.892 \\
\hline Median post-operative sCr, $\mu \mathrm{mol} / \mathrm{L}$ (IQR) & $85.7(68-96)$ & $86.1(67-93)$ & $85.8(69-97)$ & 0.951 & 0.827 \\
\hline Median post-operative $s \mathrm{~s}$ increase, $\mu \mathrm{mol} / \mathrm{L}$ (IQR) & $9.8(0.5-20)$ & $12(3.5-25)$ & $9(0.15-16)$ & 0.411 & 0.180 \\
\hline Median post-operative eGFR, $\mathrm{ml} / \mathrm{min}$ per $1.73 \mathrm{~m}^{2}$ (IQR) & $86.4(67-101)$ & $84.9(65-99)$ & $86.2(75-100)$ & 0.695 & 0.615 \\
\hline Median postoperative eGFR decrease, $\mathrm{ml} / \mathrm{min}$ per $1.73 \mathrm{~m}^{2}$ (IQR) & $12.4(0.7-25)$ & $14(4-28)$ & $9(0.1-20)$ & 0.207 & 0.063 \\
\hline$\%$ eGFR decrease, no(\%) & & & & 0.098 & 0.219 \\
\hline$\leq 25 \%$ & $187(72.5)$ & $42(72.4)$ & $49(84.5)$ & & \\
\hline $25.1-49.9 \%$ & $68(26.4)$ & $15(25.9)$ & $9(15.5)$ & & \\
\hline$\geq 50 \%$ & $3(1.1)$ & $1(1.7)$ & $0(0)$ & & \\
\hline Post-operative eGFR < 60, no (\%) & $36(14)$ & $11(19)$ & $8(13.8)$ & 0.975 & 0.452 \\
\hline \multicolumn{6}{|l|}{ Pathological characteristics } \\
\hline Median pathological tumor size, cm (IQR) & $3.3(2.3-4.1)$ & $3.1(2.1-4.1)$ & $3.2(2.05-4)$ & 0.334 & \\
\hline PSM, no (\%) & $13(5)$ & $5(8.3)$ & $0(0)$ & 0.020 & 0.022 \\
\hline Hystological subtypes & & & & 0.276 & 0.156 \\
\hline CRCC & $196(76)$ & $41(70.7)$ & $50(86.2)$ & & \\
\hline Chromophobe RCC & $8(3.19$ & $2(3.4)$ & $0(0)$ & & \\
\hline Papillary RCC & $11(4.3)$ & $1(1.7)$ & $1(1.7)$ & & \\
\hline Benign & $43(16.7)$ & $14(24.1)$ & 7 (12.1) & & \\
\hline Pathological stage & & & & 0.113 & 0.360 \\
\hline T1a & $150(69.9)$ & $36(80)$ & $41(80.4)$ & & \\
\hline $\mathrm{T} 1 \mathrm{~b}$ & $58(26.9)$ & $9(20)$ & 7 (13.7) & & \\
\hline $\mathrm{T} 2 \mathrm{a}$ & $5(2.3)$ & $0(0)$ & $1(2)$ & & \\
\hline ТЗа & $2(0.9)$ & $0(0)$ & $2(3.9)$ & & \\
\hline Fuhrman nuclear grade, no (\%)* & & & & 0.569 & 0.810 \\
\hline Grade 1 & $42(1.2)$ & $7(17.5)$ & $10(19.6)$ & & \\
\hline Grade 2 & $138(69.7)$ & $29(72.5)$ & $34(66.7)$ & & \\
\hline Grade 3 & $16(8.1)$ & $4(10)$ & 7 (13.7) & & \\
\hline Grade 4 & $2(1)$ & $0(0)$ & $0(0)$ & & \\
\hline MIC score, no (\%) & & & & 0.243 & 0.117 \\
\hline 1 point & $2(0.8)$ & $0(0)$ & $1(1.7)$ & & \\
\hline 2 points & $114(44.2)$ & $29(50)$ & $19(32.8)$ & & \\
\hline 3 points & $142(55)$ & $29(50)$ & $38(65.5)$ & & \\
\hline
\end{tabular}

Table 2.

Intra, postoperative and pathological results.

$\angle P N=$ Laparoscopic Partial Nephrectomy; RAPN = RobotAssisted Partial Nephrectomy; WIT $=$ Warm Ischemia Time $I Q R=$ Interquartile Range; $E B L=$ Estimated Blood Loss; OPN = Open Partial Nephrectomy; $s \mathrm{Cr}=$ serum Creatinine; eGFR = estimated Glomerular Filtration Rate PSM = Positive Surgical Margin; RCC = Renal Cell Carcinoma; $c R C C=$ clea Renal Cell Carcinoma; MIC= Margin, Ischemia and Complications.

* Fuhrman nuclear grading was avaible only in 198/215 RCC instances in LPN group. 
occurred in the group treated with LPN. Table 3 summarize and described intra and post-operative complications.

According with our hospital policy, the median hospitalization time was 7 days (IQR: 7-8) both for LPN and RAPN. No difference was found in post-operative sCr. LPN had a median increase of $\mathrm{s} C \mathrm{r}$ $9.8 \mu \mathrm{mol} / \mathrm{L}$ versus $9.3 \mu \mathrm{mol} / \mathrm{L}$ in RAPN (p-value: 0.411). In the group treated with LPN we observed a decrease in postoperative eGFR $\leq 25 \%$ in $72.5 \%$ (vs $84.5 \%$ ), between $25.1-49.9 \%$ in $26.4 \%$ (vs $15.5 \%$ ) and $\geq 50 \%$ in $1.1 \%$ (vs $0 \%$ ) of the patients. The only difference between LPN and RAPN was found in PSM rate, with a $5 \%$ and $0 \%$ respectively (p-value: 0.020 ).

Finally, the success of treatment defined with MIC score was higher in RAPN (65.5\%) than LPN (55\%) but it was statically not significant ( $p$-value: 0.243 ). The matched pair analysis shows the same results in terms of pre, intra and post-operative outcomes between LPN and RAPN.

Table 4 showed the Spearman correlation between clinical, intra and post-operative characteristic with MIC system. We found that median tumor size (rho: -0.170 p-value: 0.002), median PADUA score (rho: -0.179; p-value: $<0.001$ ), PADUA risk groups (rho: -0.191; p-value: < 0.001), renal rim (rho: -0.113; p-value: 0.044), renal sinus (rho: -0.154 ; p-value: 0.006), urinary collecting system (UCS) (rho: -0.170; p-value: 0.002 ) and tumor size coded as categorical variable (rho: -0.152; p-value: 0.007 ) were inversely related with MIC score system.

Table 4. Spearman correlation between clinical, intra and post-operative characteristics and MIC score system.

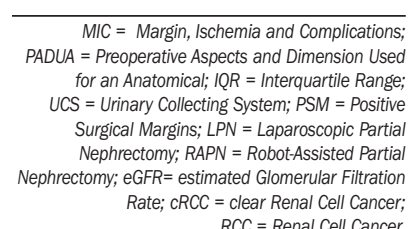

Table 3.

Intra and post-operative complications occurred (full data set).

\begin{tabular}{|l|c|c|c|c|c|}
\hline Complications & Type & Treatment & n, LPN & n, RAPN & Clavien-Dindo Grade \\
\hline Peritoneum injury & Intraoperative & Intraoperative repair & 9 & 0 & NA \\
Bleeding & Intraoperative & Blood transfusion & 6 & 1 & NA \\
Bleeding & Intraoperative & Conversion to OPN & 3 & 0 & NA \\
Spleen/Liver Injury & Intraoperative & Intraoperative repair & 0 & 2 & NA \\
Renal vein injury & Intraoperative & Intraoperative repair & 5 & 1 & NA \\
Diaphragram injury & Intraoperative & Intraoperative repair & 4 & 0 & NA \\
Fever & Postoperative & Medical therapy & 12 & 4 & 1 \\
Pain & Postoperative & Medical therapy & 10 & 2 & 1 \\
Tachycardia & Postoperative & Medical therapy & 1 & 2 & 1 \\
Atrial fibrillation & Postoperative & Medical therapy & 2 & 0 & 1 \\
Urine leak & Postoperative & Conservative menagement & 8 & 3 & 1 \\
Urine retention & Postoperative & Catheritation & 3 & 0 & 1 \\
Hb decrease & Postoperative & Blood transfusion & 8 & 4 & 2 \\
Urine leak & Postoperative & J placement & 1 & 0 & 3 \\
Hb decrease & Postoperative & Selective embolitation & 1 & 0 & 3 \\
Kidney abscess & Postoperative & Intraoperative drainage & 0 & 1 & 3 \\
\hline
\end{tabular}

LPN = Laparoscopic Partial Nephrectomy; RPN = Robot-Assisted Partial Nephrectomy; OPN = Open Partial Nephrectomy; $H b=$ Hemoglobin; $N A=$ Not Applicable

\begin{tabular}{|c|c|c|c|c|c|}
\hline \multirow[t]{2}{*}{ Variable } & & MIC & & Rho & P-value \\
\hline & $\begin{array}{l}1 \text { point } \\
(n=3)\end{array}$ & $\begin{array}{l}2 \text { points } \\
(n=133)\end{array}$ & $\begin{array}{l}3 \text { points } \\
(n=180)\end{array}$ & & \\
\hline Median age, years (IQR) & $65(57-70.5)$ & $48(40-57)$ & $50(42-59)$ & 0.044 & 0.437 \\
\hline Median clinical tumor size, cm (IQR) & $4.02(3.24-4.25)$ & $3.38(2.4-4.3)$ & $2.93(2-3.6)$ & -0.170 & 0.002 \\
\hline Median PADUA score, no (IQR) & $8.6(7-10)$ & $8.9(8-10)$ & $8.2(7-10)$ & -0.179 & $<0.001$ \\
\hline PADUA risk groups, no (\%) & & & & -0.191 & $<0.001$ \\
\hline Low & $1(33.3)$ & $29(21.8)$ & $76(42.2)$ & & \\
\hline Intermediate & $1(33.3)$ & $54(40.6)$ & $58(2.2)$ & & \\
\hline High & $1(33.3)$ & $50(37.6)$ & $46(25.6)$ & & \\
\hline Longitudinal polar location, no (\%) & & & & -0.089 & 0.115 \\
\hline Superior/inferior & $1(33.3)$ & $79(59.4)$ & $120(66.7)$ & & \\
\hline Middle & $2(66.7)$ & $54(40.6)$ & $60(33.3)$ & & \\
\hline Exophytic rate, no (\%) & & & & -0.015 & 0.793 \\
\hline$\geq 50 \%$ & $3(100)$ & $63(47.4)$ & $94(52.2)$ & & \\
\hline$<50 \%$ & $0(0)$ & $53(39.8)$ & 65 (36.1) & & \\
\hline Endophytic & $0(0)$ & $17(12.8)$ & $21(11.7)$ & & \\
\hline Renal Rim, no (\%) & & & & -0.113 & 0.044 \\
\hline Lateral & $1(33.3)$ & $73(54.9)$ & $117(65)$ & & \\
\hline Medial & $2(66.7)$ & $60(45.1)$ & $63(35)$ & & \\
\hline Renal sinus, no (\%) & & & & -0.154 & 0.006 \\
\hline Not involved & $2(66.7)$ & $87(65.4)$ & $143(79.4)$ & & \\
\hline Involved & $1(33.3)$ & $46(34.6)$ & $37(20.6)$ & & \\
\hline UCS, no (\%) & & & & -0.170 & 0.002 \\
\hline Not involved & $1(33.3)$ & 39 (29.3) & $84(46.7)$ & & \\
\hline Infiltrated/dislocated & $2(66.7)$ & $94(70.7)$ & $96(53.3)$ & & \\
\hline Tumor size & & & & -0.152 & 0.007 \\
\hline$\leq 4$ & $2(66.7)$ & $91(68.4)$ & $149(82.8)$ & & \\
\hline 4.1-7 & $1(33.3)$ & 39 (29.3) & $28(5.6)$ & & \\
\hline$\geq 7$ & $0(0)$ & $3(2.3)$ & $3(1.7)$ & & \\
\hline Median WIT, min (IQR) & $27.2(26-30)$ & $25.5(22-29)$ & $16.8(15-20)$ & -0.672 & $<0.001$ \\
\hline Clavien Dindo complication, no (\%) & & & & -0.137 & 0.015 \\
\hline Minor & $0(0)$ & $26(19.5)$ & $33(18)$ & & \\
\hline Major & $2(66.7)$ & $1(0.8)$ & $0(0)$ & & \\
\hline PSM, no (\%) & $1(33.3)$ & $12(9)$ & $0(0)$ & -0.256 & $<0.001$ \\
\hline Surgical tecnique & & & & 0.072 & 0.204 \\
\hline LPN & $2(66.7)$ & $114(85.7)$ & $142(79.9)$ & & \\
\hline RAPN & $1(33.3)$ & $19(14.3)$ & $38(21.1)$ & & \\
\hline eGFR, no (\%) & & & & & \\
\hline eGFR $<60 \mathrm{ml} / \mathrm{min}$ per $1.73 \mathrm{~m}^{2}$ & $1(33.3)$ & $29(21.8)$ & $14(7.8)$ & 0.206 & $<0.001$ \\
\hline eGFR $\geq 60 \mathrm{ml} / \mathrm{min}$ per $1.73 \mathrm{~m}^{2}$ & $2(66.7)$ & $104(78.2)$ & $166(92.2)$ & & \\
\hline Median Fuhrman nuclear grade, (IQR) & $2(2-3)$ & $1.8(2)$ & $1.8(2)$ & -0.144 & 0.023 \\
\hline Pathological stage, no (\%) & & & & -0.111 & 0.069 \\
\hline $\mathrm{T} 1 \mathrm{a}$ & $1(33.3)$ & $76(67.9)$ & $117(76)$ & & \\
\hline $\mathrm{T} 1 \mathrm{~b}$ & $1(33.3)$ & $31(27.7)$ & $33(21.4)$ & & \\
\hline $\mathrm{T} 2 \mathrm{a}$ & $1(33.3)$ & $3(2.7)$ & $2(1.3)$ & & \\
\hline T3a & $0(0)$ & $2(1.8)$ & $2(1.3)$ & & \\
\hline Histologic subtypes, no (\%) & & & & -0.005 & 0.932 \\
\hline $\mathrm{cRCC}$ & $2(66.7)$ & $104(78.2)$ & $140(77.8)$ & & \\
\hline Chromophobe RCC & $0(0)$ & $2(1.5)$ & $6(3.3)$ & & \\
\hline Papillary RCC & $1(33.3)$ & $4(3)$ & $7(3.9)$ & & \\
\hline Benign & $0(0)$ & $23(17.3)$ & $27(15)$ & & \\
\hline
\end{tabular}


Table 5.

Independent factors related with MIC success: multivariable analysis.

\begin{tabular}{|l|c|c|c|}
\hline Variable & p-value & OR & $\mathbf{9 5 \%} \mathbf{~ C l}$ \\
\hline Clinical tumor size & 0.035 & 0.829 & $0.697-0.987$ \\
\hline PADUA score (continuously coded) & $<0.001$ & 0.843 & $0.740-0.960$ \\
\hline PADUA risk group & $<0.001$ & reference & \\
$6-7$ & $<0.001$ & 0.416 & $0.238-0.729$ \\
$8-9$ & $<0.001$ & 0.356 & $0.199-0.636$ \\
$\geq 10$ & $<0.001$ & 0.598 & $0.530-0.675$ \\
\hline WIT & & & \\
\hline Post operative eGFR level & & \\
eGFR $<60 \mathrm{ml} /$ min per $1.73 \mathrm{~m}^{2}$ & $<0.001$ & reference & \\
eGFR $\geq 60 \mathrm{ml} /$ min per $1.73 \mathrm{~m}^{2}$ & $<0.001$ & 3.356 & $1.701-6.621$ \\
\hline Fuhrman nuclear grade & 0.014 & 1.798 & $1.129-2.865$ \\
\hline
\end{tabular}

$O R=$ Odds Ratio; $\mathrm{Cl}=$ Confidence Interval; $P A D U A=$ Preoperative Aspect and Dimension Used for an Anatomical; eGFR = estimated Glomerular Filtration Rate

As aspect, the strongest factor related with MIC score system was WIT (rho: -0.672; p-value: <0.001). The surgical technique (LPN vs RAPN) was not statically related with MIC in this report (p-value: 0.204). Clavien Dindo complications and PSM were inversely related with MIC score system (rho: -0.137 and -0.256; p-value: 0.015 and $<0.001$ respectively). Two important aspects we found in this analysis. An eGFR level $\geq 60 \mathrm{ml} / \mathrm{min}$ per $1.73 \mathrm{~m}^{2}$ (rho: 0.206; p-value: <0.001) and Fuhrman nuclear grade (rho: -0.144; p-value: 0.023) were related with MIC. Table 5 showed binary logistic regression analysis reporting independent factors related with MIC success (WIT $\leq 20$ minutes, no major complications, no PSM). Clinical tumor size (p-value: < 0.001; OR: 0.829; 95\% CI: 0.697-0.987), PADUA score (p-value: < 0.001; OR: 0.843; 95\% CI: 0.740-0.960), PADUA risk groups (low: reference; intermediate; p-value: < 0.001; OR: 0.416; 95\% CI: 0.238 0.792; high: p-value: <0.001; OR: 0.356; 95\% CI: 0.199 0.636), WIT (p-value: < 0.001; OR: 0.598; 95\% CI: $0.530-0.675)$ were independently related with MIC success. eGFR ( $\geq 60 \mathrm{vs}<60 \mathrm{ml} / \mathrm{min}$ per $1.73 \mathrm{~m}^{2}$ : p-value: $<0.001$; OR: 3.356; 95\% CI: 1.701-6.621) and Fuhrman nuclear grade (p-value: 0.014; OR: 1.798; 95\% CI:1.1292.865) were also independent factors.

\section{Discussion}

The findings of this study shows that MIC score system is a simple and useful tool to report and compare different surgical approach. We did not find any difference in clinical, intra and post-operative outcomes between LPN and RAPN. Clinical tumor size, PADUA score, PADUA risk groups and WIT were independently associated with MIC. Several Authors (8) recently proposed a score system to evaluate partial nephrectomy based on WIT $\leq 20$ minutes, negative SM and no major complications. This system is similar to the trifecta outcomes proposed and validated by other groups of Authors (20-21). Hung et al (21), definited the trifecta outcomes when there was negative SM, minimal renal function decrease and no urological complications. Khalifeh et al. (20), definited trifecta outcomes as a WIT $\leq 25$ minutes, negative SM and no intra and post-operative complications. Recently, Minervini et al. (22) validated the trifecta outcomes in a matched-pair comparison between OPN and LPN in clinical Tla renal mass. The MIC system (8) is based on aspects validated by literature. Recently a panel of experts proposed that WIT should not ideally exceed 20 minutes 4 and every minute counts when the hilum is clamped (23). The Clavien-Dindo classification is the most validated tool to standardized and report surgical complications. Mottrie (8) defined the MIC score system simple to use and encouraged new research to assess is efficacy, especially by comparing its use in different surgical approaches (OPN, LPN and RAPN). Porpiglia (24) was the first to assess the learning curve in LPN using this scoring system. He divided his experience in 4 eras and noted an increase of MIC along the learning curve. In the current study we reported a MIC rate of 55\% and $65.5 \%$ in LPN and RAPN respectively. We evaluated RAPN performed in the last year after the learning curve was completed (7) and the approach was standardized. In this report, LPN and RAPN groups had similar clinical, intra and post-operative characteristics. In a recent meta-analysis (25) LPN and RAPN did not show any difference in operative time, EBL, hospital stay, oncological results and postoperative outcomes, but RAPN had a shorter WIT than LPN. In the current study the median WIT was similar between the two groups (20.8 vs 19.4 minutes) and this results are in line with the literature ( 7 , 12, 25). Ficarra et al. (26), in a multicenter study, reported a median WIT of 16 for low-risk tumors in RAPN and Porpiglia et al. (24), showed that WIT decreases along the learning curve in LPN with results comparable to OPN and RAPN. A recent study (13) showed that there was no difference between RAPN and LPN in complex tumors (median renal score 8), and this was explained by the Authors on the basis of experienced surgeon's experience in laparoscopic and robotic surgery in high volume centers. The transition from LPN to RAPN is simple and can be associated with immediate improvements in perioperative parameters for surgeons with a solid baseline experience with LPN. RAPN may reduce the technical difficulties of LPN (7), especially in complex cases, but a laparoscopic skills are important in robotic surgery. The absence of PSM reported in our RAPN cohort, described the oncological safety of RPN (7). Negative SM is the first goal of PN, and a combination of a highly malignant tumor with PSM seems to increase the risk of local recurrence (24). Another important aspect of this study is the evaluation of MIC score. PN is a complex procedure that require several aspects to assess the success. MIC represents a good tool to assess the success of PN. Tumor's anatomical characteristics were related with MIC success. As reported by Porpiglia (24), MIC rate was higher in low risk groups. Our study efforts this theory. MIC score system was inversely related with PADUA score and clinical tumor size. Interestingly we found a correlation between post-operative eGFR level $\geq 60 \mathrm{ml} / \mathrm{min}$ per $1.73 \mathrm{~m}^{2}$ and Furhman nuclear grade. This finding might be explained by the influence of WIT on renal function and by the aggressiveness of the tumor assessed by the Furhman nuclear grade. This finding needs further evaluations. This is a retrospective single center single surgeon study. This study has several limitations; long-term oncological follow-up was not reported in this series. The LPN group was bigger than RAPN group. We report- 
ed the RAPN performed in the last year after the learning curve was completed ( $>30$ cases). PN was performed by retroperitoneal approach in laparoscopic group and by transperitoneal approach in robotic groups. In our institute the retroperitoneal approach is the standardize methods for laparoscopic kidney surgery, both for posterior and anterior masses.

The matched-pair analysis was performed in order to evaluate if the different number of patients into the two groups can alter our statistical results.

Other Authors (22) used this method to compare different groups. We set the matched-pair analysis on 1:1 ratio in order to obtain two similar groups. The matched pair analysis was based on the tumor characteristics (size and PADUA score). We found no differences also in the matched pair analysis. The full data set was used in the Spearman correlation and logistic regression in order to didn't have overfitting problems. Another important limitation is a short follow-up for evaluating kidney function (median 30 days) assessed only by eGFR. MDRD equation has limitations for eGFR evaluation. $\mathrm{s} C \mathrm{r}$ is the best predictor of eGFR in MDRD equation, but his levels are impacted by BMI, gender, ethnicity, age and hydratation status.

\section{Conclusion}

Our report showed that the MIC score system is simple and useful to report and compare different surgical approach. The use of nephrometry score system, as reported by other Authors (26-27), is useful to predict outcomes after partial nephrectomy. The MIC score is influenced by several anatomical aspects and the use of nephrometry score is useful to predict MIC success. From our experience, we believe that MIC system could be a helpful tool to assess PN outcomes and to compare different surgical approach.

\section{References}

1. Ljungberg B, Cowan NC, Hanbury DC, et. EAU guidelines on renal cell carcinoma: the 2010 update. Eur Urol. 2010; 58:398-406.

2. Novick AC, Campbell SC, Belldegrun A, et al. Guideline for management of the clinical stage 1 renal mass. American Urological Association Web site. http://www.auanet.org/content/media/renal mass09.pdf

3. Van Poppel H, Da Pozzo L, Albrecht W, et al. A prospective randomized EORTC intergroup phase 3 study comparing the complications of elective nephron-sparing surgery and radical nephrectomy for low-stage renal cell carcinoma. Eur Urol. 2007; 51:1606-15.

4. Becker F, Van Poppel H, Hakenberg OW, et al. Assessing the impact of ischaemia time during partial nephrectomy. Eur Urol. 2009; 56:625-34.

5. Pignot G, Bouliere F, Patard JJ. Warm ischaemia: the ultimate enemy for partial nephrectomy? Eur Urol. 2010; 58:337-9.

6. Gettman MT, Blute ML, Chow GK, Neururer R, et al. Roboticassisted laparoscopic partial nephrectomy: technique and initial clinical experience with DaVinci robotic system. Urology. 2004; 64:914-8.

7. Mottrie A, De Naeyer G, Schatteman P, et al. Impact of the learn- ing curve on perioperative outcomes in patients who underwent robotic partial nephrectomy for parenchymal renal tumours. Eur Urol. 2010; 58:127-33.

8. Buffi N, Lista G, Larcher A, et al. Margin, ischemia, and complications (MIC) score in partial nephrectomy: a new system for evaluating achievement of optimal outcomes in nephron-sparing surgery. Eur Urol. 2012; 62:617-8.

9. Dindo D, Demartines N, Clavien PA. Classification of surgical complications: a new proposal with evaluation in a cohort of 6336 patients and results of a survey. Ann Surg. 2004; 240:205-13.

10. Zhang X, Li HZ, Ma X, et al. Retroperitoneal laparoscopic nephron-sparing surgery for renal tumors: report of 32 cases. Urology. 2005; 65:1080-4.

11. Zhang X, Fu B, Lang B, et al. Technique of anatomical retroperitoneoscopic adrenalectomy with report of 800 cases. J Urol. 2007; 177:1254-7.

12. Benway BM, Bhayani SB, Rogers CG, et al. Robot-assisted partial nephrectomy: an international experience. Eur Urol. 2010; 57:815-20.

13. Long JA, Yakoubi R, Lee B, et al. Robotic versus laparoscopic partial nephrectomy for complex tumors: comparison of perioperative outcomes. Eur Urol. 2012; 61:1257-62.

14. Ficarra V, Novara G, Secco S, et al. Preoperative aspects and dimensions used for an anatomical (PADUA) classification of renal tumors in patients who are candidates for nephron-sparing surgery. Eur Urol. 2009; 56:786-93.

15. Reporting and grading of complications after urologic surgical procedures: an ad hoc EAU guidelines panel assessment and recommendations. Mitropoulos D, et al.; European Association of Urology Guidelines Panel. Eur Urol. 2012; 61:341-9.

16. Levey AS, Bosch JP, Lewis JB, et al. A more accurate method to estimate glomerular filtration rate from serum creatinine: a new prediction equation. Modification of Diet in Renal Disease Study Group. Ann Int Med. 1999; 130:461-70.

17. Eble JN, Sauter G, Epstein JI, et al., editors. Pathology and genetics of tumors of the urinary system and male genital organs. World Health Organization classification of tumors. Lyon, France: IARC Press; 2004.

18. Greene FL, Gospodarowicz M, Wittekend C, et al. American Joint Committee on Cancer (AJCC) staging manual. ed. 7. Philadelphia, PA: Springer; 2009.

19. Fuhrman S, Lasky LC, Limas L. Prognostic significance of morpho- logic parameters in renal cell carcinoma. Am J Surg Pathol 1982; 6:655-63.

20. Khalifeh A, Autorino R, Hillyer SP, et al. Comparative outcomes and assessment of trifecta in 500 robotic and laparoscopic partial nephrectomy cases: a single surgeon experience. J Urol. 2013; 189:1236-42.

21. Hung AJ, Cai J, Simmons MN, Gill IS. "Trifecta" in partial nephrectomy. J Urol. 2013; 189:36-42.

22. Minervini A, Siena $G$, Antonelli A, et al. Open versus laparoscopic partial nephrectomy for clinical Tla renal masses: a matchedpair comparison of 280 patients with TRIFECTA outcomes (RECORd Project). WJ Urol. 2014; 32:257-63.

23. Thompson RH, Lane BR, Lohse CM, et al. Every minute counts when the renal hilum is clamped during partial nephrectomy. Eur Urol. 2010; 58:340-5.

24. Porpiglia F, Bertolo R, Amparore D, Fiori C. Margins, ischaemia 
and complications rate after laparoscopic partial nephrectomy: impact of learning curve and tumour anatomical characteristics. BJU Int. 2013; 112:1125-32.

25. Aboumarzouk OM, Stein RJ, Eyraud R, et al. Robotic versus laparoscopic partial nephrectomy: a systematic review and metaanalysis. Eur Urol. 2012; 62:1023-33.

26. Ficarra V, Bhayani S, Porter J, et al. Predictors of warm ischemia time and perioperative complications in a multicenter, international series of robot-assisted partial nephrectomy. Eur Urol. 2012; 61:395402.

27. Sea JC1, Bahler CD, Mendonsa E, et al. Comparison of measured renal tumor size versus R.E.N.A.L. nephrometry score in predicting patient outcomes after robot-assisted laparoscopic partial nephrectomy. J Endourol. 2013; 27:1471-6.

\section{Correspondence}

Stefano Ricciardulli, MD (Corresponsing Author) stefano.ricciardulli@gmail.com

Department of Urology, San Bassiano Hospital

Via dei Lotti 40 - Bassano del Grappa, Italy

Department of Urology, Chinese PLA General Hospital

No. 28 Fuxing Road, Beijing 100853, China

Quiang Ding, MD

dingqiangchina@foxmail.com

Xu Zhang PhD, MD (Corresponding Author)

xzhang@foxmail.com

Hongzhao Li, MD

urolancet@126.com

Yuzhe Tang, MD

townyuzhe@gmail.com

Guoqiang Yang, MD

gqyang@outlook.com

Xiyou Wang, MD

510198870@qq.com

Xin $M a, M D$

Department of Urology, Chinese PLA General Hospital

No. 28 Fuxing Road, Beijing 100853, China

urologist@foxmail.com

Alberto Breda, MD

albbred@hotmail.com

Department of Urology, Foundacio Puigvert

Universidad Autonoma de Barcelona

Barcelona (Spain)

Antonio Celia, MD

antonio.celia@aslbassano.it

Department of Urology, San Bassiano Hospital

Bassano del Grappa, Italy 